\title{
Psoas abscess during treatment with intravenous tocilizumab in a patient with rheumatoid arthritis: a case-based review
}

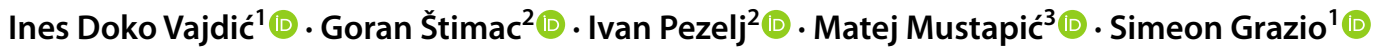

Received: 18 January 2021 / Accepted: 22 January 2021 / Published online: 12 February 2021

(C) The Author(s), under exclusive licence to Springer-Verlag GmbH, DE part of Springer Nature 2021

\begin{abstract}
Interleukin-6 receptor antagonist tocilizumab is a biologic drug used for treating patients with active rheumatoid arthritis (RA) who failed to respond to synthetic or other biologic disease-modifying antirheumatic drugs or where they were contraindicated. Interleukin- 6 receptor blockade results in a decrease of disease activity but has some potential adverse effects, the most common being infections. We present a case of a 75-year-old female patient with long-lasting RA, several comorbidities and multiple prior therapies, who developed back pain and general malaise during tocilizumab intravenous treatment. The laboratory findings were typical of toxemia, and the imaging findings revealed large psoas muscle abscess. Surgical and antibiotic treatment was performed with a good outcome. To our knowledge, this has been the first case of a psoas abscess in a patient with RA treated with tocilizumab described in the literature so far. We also present a review of the literature regarding infection, and particularly abscess formation in patients treated with biological disease-modifying antirheumatic drugs, tocilizumab included.
\end{abstract}

Keywords Abscess $\cdot$ Psoas $\cdot$ Tocilizumab $\cdot$ Arthritis $\cdot$ Rheumatoid $\cdot$ Diagnosis $\cdot$ Treatment

\section{Introduction}

Simeon Grazio

simeon.grazio@zg.t-com.hr

Ines Doko Vajdić

inesdoko@yahoo.com

Goran Štimac

gogostimac@gmail.com

Ivan Pezelj

pezelj.ivan@gmail.com

Matej Mustapić

mmustapicm@gmail.com

1 University Department of Rheumatology, Physical and Rehabilitation Medicine, School of Medicine Zagreb, University Hospital Centre Sestre Milosrdnice, Vinogradska 29, 10000 Zagreb, Croatia

2 University Department of Urology, University Hospital Centre Sestre Milosrdnice, Vinogradska 29, 10000 Zagreb, Croatia

3 Department of Radiology, Sahlgrenska University Hospital Gothenburg and Southern Älvsborg Hospital Borås, Borås, Sweden
Interleukin-6 (IL-6) plays an important role in the pathogenesis of rheumatoid arthritis (RA). Tocilizumab, a monoclonal antibody against IL-6 receptor, prevents the binding of IL-6 to its receptor and blocks the signal [1]. It is indicated in patients with active RA who failed to respond to conventional synthetic or other biologic disease-modifying antirheumatic drugs (DMARDs) [2, 3]. It has a beneficial effect on both articular and systemic levels [1]. The most common side effects of tocilizumab are an increased risk of infection, especially bacterial, hypercholesterolemia, and diverticulitis [4].

An abscess may be either skin or internal, although the former is more common. A notion of it being a serious condition is supported by the data that in the United States in 2005 there were 3.2 million emergency department admissions for an abscess [5], while in Australia, in 2008, around 13,000 people with this condition were hospitalized [6].

A psoas abscess is a rare condition and the reported incidence worldwide is 12 cases per year [7]. It occurs by spreading of the infection from a distant focus (lymphogenic or hematogenous) - a primary psoas abscess, or by spreading directly from a nearby location (e.g. the urinary system) 
to the psoas muscle sheath-a secondary psoas abscess [7]. Common microbiological causative agents are Staphylococcus aureus, Escherichia coli, Bacteroides species and Mycobacterium tuberculosis [8]. A primary psoas abscess is more common in HIV-positive individuals and children, while a secondary form is more common in diabetics, patients on glucocorticoid therapy and patients with renal failure. A secondary psoas abscess, which is more prevalent, may have its origin in the gastrointestinal, urogenital, musculoskeletal or other nearby organ systems. Within the musculoskeletal system, the most common entities are spondylodiscitis, osteomyelitis, septic arthritis, and sacroiliitis [9]. Here, we present a case of a psoas abscess in a patient with RA treated with intravenous tocilizumab, along with the review of the literature on the topic. Prior to collecting and analysing the data, and executing this work, written consent was obtained from the patient.

\section{Case report}

A 75-year-old female patient with long-standing RA treated with intravenous tocilizumab, was admitted in the emergency department because of the pain in the left lumbar region, accompanied by a fever and vomiting. Her history revealed that she had been diagnosed with RA 35 years ago. As for the conventional synthetic DMARDs for RA, she had previously been treated with sulfasalazine and chloroquine, which were discontinued due to ineffectiveness, followed by methotrexate (both orally and intramuscular) and leflunomide, which were discontinued due to side effects. Thereafter, the patient was treated with tumor necrosis factor alpha (TNF- $\alpha$ ) inhibitor adalimumab for 2 years, which was discontinued due to secondary inefficiency. She had aloarthroplasty of the left knee 13 years ago with a good functional outcome. As for comorbidities, the patient had coronary heart disease, hypertension, hyperlipidaemia, osteoporosis and multiple herniations of thoracic and lumbar intervertebral discs, the latter being a possible reason for uncertainties regarding the diagnosis at the onset of the symptoms.

Symptoms suggesting urinary infection (discomfort during voiding, bladder pain and cloudy urine) started 3 weeks earlier and the microbiological analysis showed E. coli in significant number $\left(10^{5} \mathrm{CFU} / \mathrm{ml}\right)$. She was treated with different oral antibiotics in sequential way (according to the antibiogram, starting with norfloxacin followed by amoxicillin + clavulanic acid), as well as with analgesics, including intramuscular administration, both prescribed by her general practitioner resulting in a mild improvement in symptoms. The pain in the lumbar region began 2 weeks prior to her admission, as well as loss of appetite and loss of body weight (6 kg in total).
For her RA the patient had been receiving tocilizumab (680 mg IV) once a month for the previous 30 months, with no interruptions, in combination with an orally administered low-dose prednisone ( $5 \mathrm{mg}$ per day). She experienced an excellent clinical response, with a decrease from the baseline to the presented moment in DAS28 (from 7.27 to 4.759), and HAQ (from 2.25 to 1.875 ).

At the admission, she was presented as an obese (BMI $32.4 \mathrm{~kg} / \mathrm{m}^{2}$ ), weary and tired ambulating female patient, with a fever $\left(38.5^{\circ} \mathrm{C}\right)$ and tenderness during palpation of the left lumbar region. Also, typical signs of chronic RA (thickening and flexion contracture of metacarpophalangeal joints, ulnar deviation of fingers of both hands, flexion contracture of the right elbow, thickening, soreness and flexion contracture of the right knee) and chronic low-back pain (decreased range of motion with slight pain at the end of movements, but no radiating pain into legs) were observed, too.

As for the laboratory tests, there were spiking values in the following results: highly increased C-reactive protein $(149.4 \mathrm{mg} / \mathrm{L})$ and erythrocyte sedimentation rate $(105 \mathrm{~mm} / 3.6 \mathrm{ksec})$, leucocytosis $\left(11.8 \times 10^{9} / \mathrm{L}\right)$ with neutrophilia (77.9\%), and anaemia (haemoglobin $98 \mathrm{~g} / \mathrm{L}$ ). Also, urine analysis revealed leukocyturia and bacteriuria (more than 100 leukocytes per power field). The abdomen ultrasound exam showed an inhomogeneous, partly solid and partly liquid formation next to the lower pole of the left kidney (Fig. 1). An abdominal computerized tomography (CT) scan (Fig. 2a, b) was also performed showing a collection of fluid sized $10 \times 10 \times 17 \mathrm{~cm}$ in the left retroperitoneal region, in the area of the psoas muscle. The MRI (magnetic resonance imaging) of the lumbar spine confirmed a psoas abscess. Tocilizumab was immediately excluded from the therapy, and prednisolone was tapered quickly off until exclusion. The patient underwent extraperitoneal surgical drainage of the abscess, from which $E$. coli was isolated.

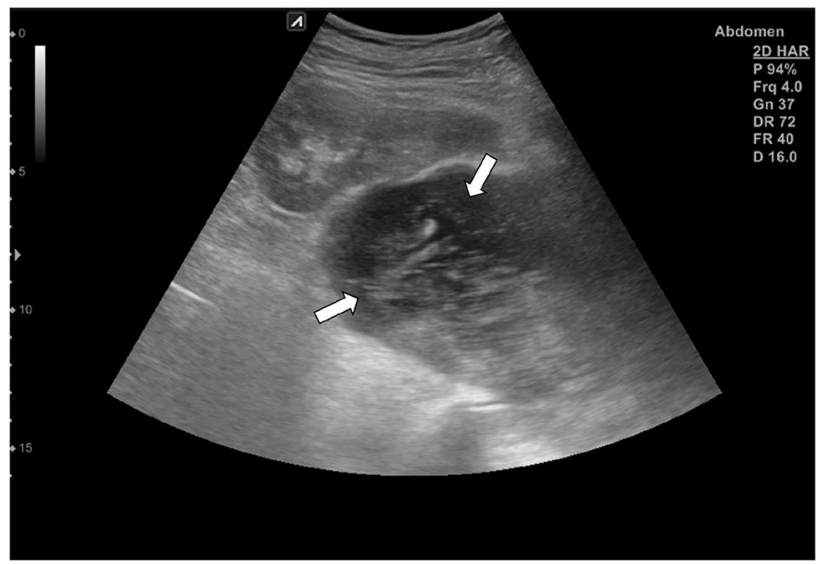

Fig. 1 Abdominal ultrasonography: inhomogeneous formation next to the lower pole of the left kidney, partly solid and partly liquid (arrows) 
Fig. 2 a Abdominal CT scan-coronal view: a well circumscribed retroperitoneal collection of dense liquid and partially gaseous contents (arrows) measuring $10 \times 10 \times 17$ $\mathrm{cm}$ in projection of the left psoas muscle. b Abdominal CT scan-axial view: a collection of dense liquid and partially gaseous contents (arrows) within and along the left psoas muscle
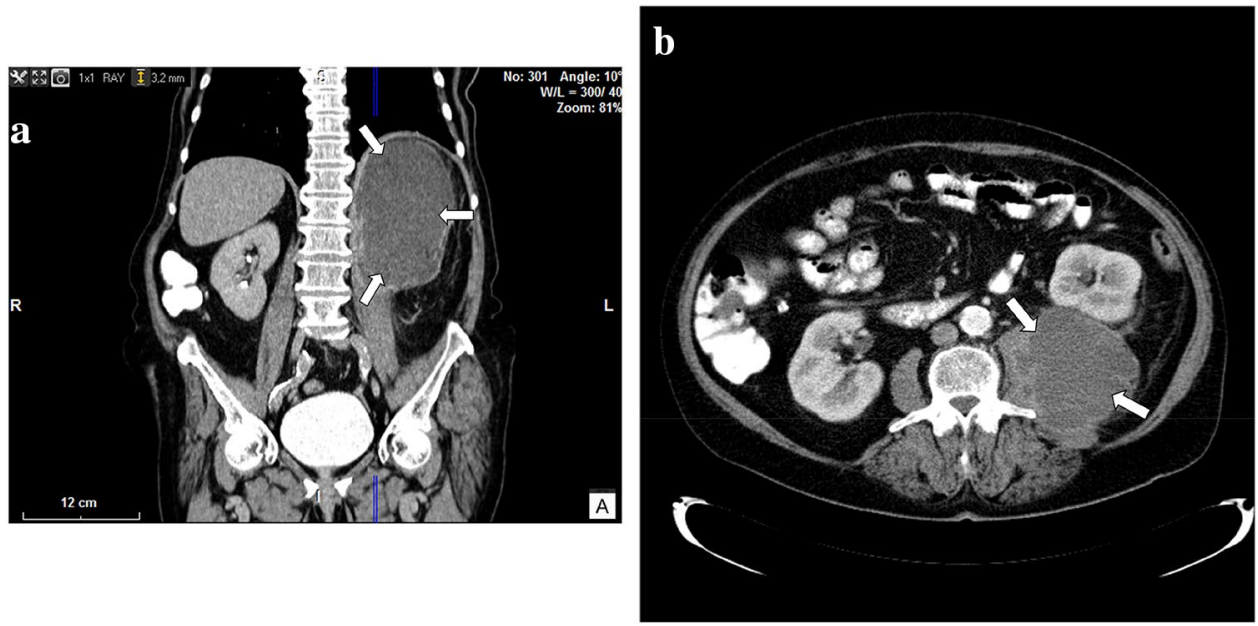

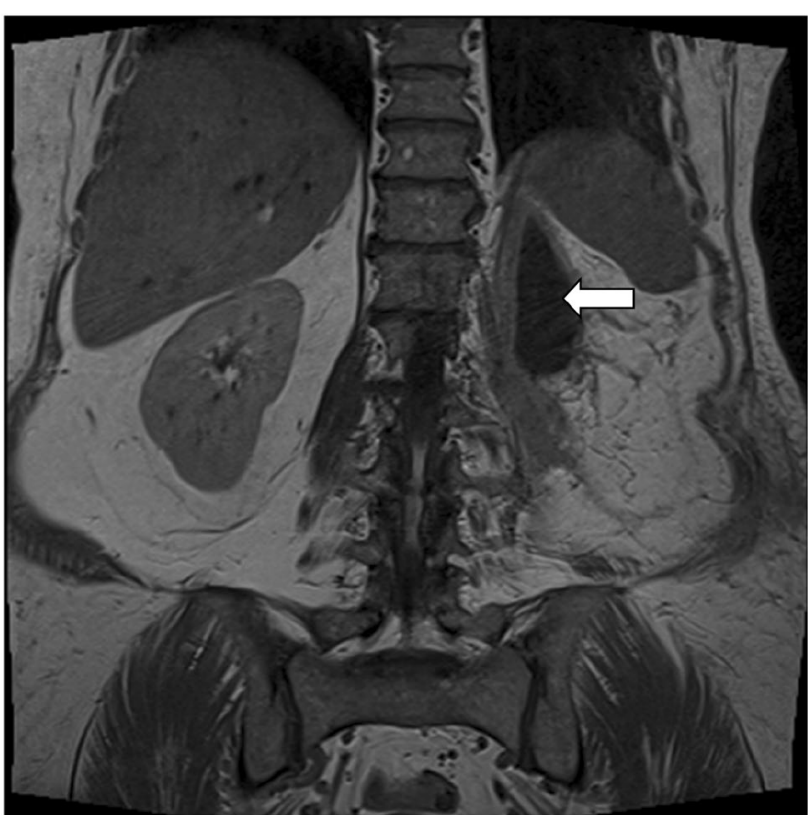

Fig. 3 Control MRI: an abscess collection within the left psoas muscle (arrow), measuring $5 \times 7 \mathrm{~cm}$, with surrounding inflammation of adipose tissue

Also, a double parenteral antimicrobial therapy was applied (co-amoxiclav and gentamicin). This resulted in the disappearance of the symptoms and abscess regression on imaging (Fig. 3). After that, the patient continued to take amoxicillin with clavulanic acid at a dose of $1 \mathrm{~g}$ twice a day orally for 5 days, followed by 10 days of $500 \mathrm{mg}$ ciprofloxacin twice a day. Since then the patient was without any signs or symptoms of the recurrence of the psoas abscess. She is under regular check-ups by urologists and nephrologists for recurrent urinary tract infections, which she is obviously prone to, and on one occasion she was hospitalized for acute pyelonephritis, successfully treated according to the antibiogram.
As for RA, due to unclear data from the medical records on the side effect of previous use of leflunomide, and in shared decision making with the patient, re-administration of leflunomide at a dose of $20 \mathrm{mg}$ was attempted. The patient did not develop side effects and the drug was well-tolerated, but she is still dependent on a low-dose prednisolone with insufficient disease control. Respecting the patient's age and comorbidities, the introduction of a targeted synthetic DMARD is being considered. In the meantime, minimally invasive treatment for L4-L5 disc herniation with a good outcome was performed.

\section{Search strategy}

We searched the PubMed and Scopus databases for original articles, reviews, letters, short communications and notes in English from 1971 for PubMed and from 2004 for Scopus to the latest date for both of them of October 19th 2020. Our search strategy included looking for titles, abstracts, and keywords using the following default settings: "infection" AND "biological disease-modifying antirheumatic drugs" AND "rheumatoid arthritis", "infection" AND "tocilizumab" AND "rheumatoid arthritis", "urinary infection" AND "tocilizumab" AND "rheumatoid arthritis", "abscess" AND "biological disease-modifying drugs", "abscess" AND “tocilizumab”, "abscess" AND ,tocilizumab" AND "rheumatoid arthritis". The search results are shown in Table 1 [10].

\section{Discussion}

Our patient suffering from long-term RA on tocilizumab monotherapy and concomitant low dose of glucocorticoids developed a psoas abscess as a complication of urinary tract infection. Previous studies have shown an association 
Table 1 Search strategy of the literature

\begin{tabular}{lcr}
\hline Search terms and Boolean operators & \multicolumn{2}{l}{ Number of publications } \\
\cline { 2 - 3 } & PubMed & Scopus \\
\hline $\begin{array}{l}\text { "Infection" AND "biologic disease-modifying antirheumatic drugs" AND } \\
\text { "rheumatoid arthritis" }\end{array}$ & 11 & 64 \\
"Infection" AND "tocilizumab" AND "rheumatoid arthritis" & 167 & 1263 \\
"Urinary infection" AND "tocilizumab" AND "rheumatoid arthritis" & 0 & 0 \\
"Abscess" AND "biologic disease-modifying drugs" & 0 & 0 \\
"Abscess" AND "tocilizumab" & 6 & 94 \\
"Abscess" AND "tocilizumab" AND "rheumatoid arthritis" & 2 & 0 \\
\hline
\end{tabular}

between older age ( $>65$ years), disease duration ( $>10$ years), and concomitant glucocorticoid therapy ( $>5 \mathrm{mg}$ daily) with the development of serious infections during tocilizumab treatment [11, 12].

The safety and efficacy of tocilizumab in monotherapy have been elucidated in detail in a meta-analysis by Nishimoto et al. [13]. The results showed that infections, followed by gastrointestinal disorders and changes in the skin and subcutaneous tissue are the most common side effects. Changes in laboratory findings, such as dyslipidemia and elevated liver enzymes, are also common. As for infections, the most common are mild infections of the upper respiratory tract, while the most prominent serious infections are pneumonia, herpes zoster and cellulitis. It is important to note that the frequency of infections was not related to the duration of tocilizumab administration. No significant differences in the safety profile were observed between the recently introduced subcutaneous IL-6 receptor blocker sarilumab and tocilizumab [14].

In a head-to-head comparison of the safety of tocilizumab and TNF- $\alpha$ inhibitors in Japanese patients, a higher frequency of concomitant use of oral glucocorticoids was observed in the tocilizumab group. A higher incidence of serious infections has also been reported in patients treated with tocilizumab who were concomitantly taking oral glucocorticoids at a dose equivalent to $5 \mathrm{mg}$ of prednisolone daily or higher [15]. Our patient was treated with such a combination.

Due to nonspecific symptoms and diverse clinical features, a psoas abscess can be mistaken for diverticulitis, retrocecal appendicitis, a psoas muscle hematoma, iliopsoas bursitis, sciatica, metastatic disease or even aortic aneurysm [9]. Infectious spondylodiscitis could have also been suspected in our patient, due to the previously described increased risk of hematogenous infection of degeneratively altered intervertebral discs. In the case reported by Tsujimoto et al., the patient presented with almost the same symptoms (low back pain, fatigue, fever and dyspnoea), and highly elevated CRP values $(133 \mathrm{mg} / \mathrm{L})$ as in our case. The patient was also treated with a standard dose of IV tocilizumab, and a low dose of methylprednisolone (2 mg daily) for 2 years. Compared to our patient, she had a lower BMI $\left(18.6 \mathrm{~kg} / \mathrm{m}^{2}\right)$ and shorter disease duration (14 years), while as for comorbidities, cardiovascular disease was present, too [16]. A recent case report showed the occurrence of spinal cord abscesses with associated motor deficits in patients treated with tocilizumab for COVID-19 [17]. An epidural abscess with associated osteomyelitis of the spine has also been described in patients with RA treated with tocilizumab after a transient bacteriemia caused by Salmonella enteritidis [18]. A group of authors from Japan reported a case of disseminated infection and bacteriemia with Nocardia brasiliensis. The patient was treated for RA with subcutaneous tocilizumab (162 mg every 2 weeks) for 2 months, with concomitant prednisolone ( $7.5 \mathrm{mg}$ per day) and methotrexate (8 mg per week), both administered for a decade. Abscesses confirmed by a CT scan were observed in the lungs and subcutaneously in the left buttock region [19]. Also, a streptococcal lung abscess has been described in patients with Takayasu arteritis treated with tocilizumab [20].

In therapy-resistant adult-onset Still's disease, tocilizumab, along with IL-1 receptor antagonist anakinra, has been shown to be the most effective biological drug with a satisfactory safety profile. One case of a psoas abscess with Staphylococcus aureus was described but the authors did not provide the information whether concomitant immunosuppressive or immunomodulatory therapy was used [21]. Moreover, a fatal outcome was observed in a patient with streptococcal infection in the area of the pectoralis major and minor and latissimus dorsi muscles. The patient was receiving tocilizumab monotherapy for RA and died of necrotizing fasciitis complicated by sepsis [22].

It is of note that in the defence against Mycobacterium tuberculosis infection, the production of interferon gamma (INF- $\gamma$ ) is crucial. Tocilizumab has been shown to have an insignificant effect on the reduction of INF- $\gamma$. Therefore, compared to TNF- $\alpha$ inhibitors (i.e. infliximab and etanercept), it has a lower risk of tuberculosis reactivation [23].

Manabe et al. presented a case of a patient with necrotizing soft tissue infection emphasizing the importance of recognizing infections in patients treated with tocilizumab in whom the symptoms of toxemia are masked by IL-6 
blockade [24]. Tocilizumab is known to reduce the production of acute-phase reactants by its mechanism of action and thus the detection of infection may be delayed [25]. It also reduces the $\mathrm{B}$ and $\mathrm{T}$-cell response as well as cytotoxicity, which contributes to an increased susceptibility to infections in these patients [26]. Transient neutropenia can be present early after drug administration (usually within the first six weeks), but it is not yet clear whether this is associated with an increased risk of serious infections [27]. Compared to synthetic drugs, a higher risk of developing serious infections was observed in the first year of treatment with biologics. This is also associated with the degree of disease activity and concomitant therapy. Later, the mechanism of action of the drug itself becomes more important, and thus the growing need for personalized prevention of the infection risk. Various agent-specific biomarkers are used in monitoring during treatment, and the RABITT clinical index is one of the tools available to assess the risk of infection [28, 29].

It is noteworthy that patients with inflammatory rheumatic diseases themselves, without any specific treatment, have an increased risk of infection due to immune-dysregulation inherent in the pathogenesis of the disease [30]. Regarding novel treatment of RA, TNF- $\alpha$ inhibitors carry a slightly increased and mutually comparable risk of developing a serious infection. The most common infections in those patients are bacterial infections of the urinary tract, respiratory tract and skin/subcutaneous tissue [31, 32]. Among non-TNF- $\alpha$ inhibitors in treating RA, CD20+B-depleting agent rituximab appears to have the highest risk of causing infection [33]. Rituximab-induced hypogammaglobulinemia increases susceptibility to encapsulated bacteria, and treatment is also associated with the reactivation of a HBV infection [28].

The results of a large retrospective study showed that the occurrence of serious infections in patients with RA treated with biologics is influenced, among other things, by the characteristics of patients, such as disease activity and disability, comorbidities (especially lung disease), cumulative dose of glucocorticoids and number of previous conventional systemic DMARDs [34]. This should be taken into account in this case report, because our patient had all the aforementioned pre-existing risk factors for developing a serious infection. Therefore, in this case, a causal relationship of a psoas abscess with tocilizumab alone would be concluded hastily. Also, when considering a targeted synthetic molecule, such as a JAK inhibitor, one should assess the risk in regard to recurrent urinary tract infections and thrombosis within pre-existing coronary heart disease, as well as an increased risk of herpes zoster infection in people over 65 years of age $[28,35,36]$.

The novelty of this work is that we meticulously described and pointed out that an abscess, and in this case for the first time a retroperitoneal abscess, can be an adverse event of an
IL-6 inhibitor in patients with RA. This should be taken into consideration in clinical practice if relevant symptoms occur, especially since an internal abscess, such as a psoas abscess, requires early recognition and prompt medical attention. Our case was placed in the context of the literature review which was obtained using a wide range of search terms in the two most important databases in biomedical electronic search giving comprehensibility to our work. The main limitation of this work is that it encompasses only patients on a single biologic drug, used for a particular condition. Therefore, generality should be made with caution.

\section{Conclusion}

Infection is the most common serious side effect of biologic drugs, especially in patients with a long-standing disease, comorbidities and/or concomitant glucocorticoid use.

To the best of our knowledge, this is the first case of a psoas abscess in patients with RA treated with intravenous tocilizumab (standard dose) along with a low dose of prednisone. This report contributes to the body of knowledge regarding safety issues of biologic drugs, namely tocilizumab, in the treatment of patients with RA.

Author contributions Ines Doko Vajdić, Goran Štimac, Ivan Pezelj, Matej Mustapić and Simeon Grazio were involved in the study design. Simeon Grazio and Ines Doko Vajdić masterminded and contributed significantly in writing the manuscript and merited for critical revision of the manuscript. Goran Štimac, Matej Mustapić and Simeon Grazio conducted diagnosis and treatment of the patient, reviewed the results and interpreted the data. Ines Doko Vajdić and Ivan Pezelj collected data and searched database. All authors participated in the preparation of the draft and final version of the manuscript, read and approved the version to be submitted.

The participant has consented to the submission of the case report to the journal. In preparing this manuscript, the authors adhered to the CARE guidelines.

\section{Compliance with ethical standards}

Conflict of interest Ines Doko Vajdić, Goran Štimac, Ivan Pezelj, Matej Mustapić and Simeon Grazio declare no conflict of interest.

\section{References}

1. Oldfield V, Dhillon S, Plosker GL (2009) Tocilizumab: a review of its use in the management of rheumatoid arthritis. Drugs 69:609632. https://doi.org/10.2165/00003495-200969050-00007

2. Baganz L, Richter A, Kekow J, Bussmann A, Krause A, Stille C et al (2018) Long-term effectiveness of tocilizumab in patients with rheumatoid arthritis, stratified by number of previous treatment failures with biologic agents: results from the German RABBIT cohort. Rheumatol Int 38:579-587. https://doi.org/10.1007/ s00296-017-3870-7 
3. Smolen JS, Landewé RBM, Bijlsma JWJ, Burmester G, Dougados M, Kerschbaum A et al (2020) EULAR recommendations for the management of rheumatoid arthritis with synthetic and biological disease-modifying antirheumatic drugs: 2019 update. Ann Rheum Dis 79:685-699. https://doi.org/10.1136/annrh eumdis-2019-216655

4. Gabay C, Emery P, van Vollenhoven R, Dikranian A, Alten R, Pavelka K et al (2013) Tocilizumab monotherapy versus adalimumab monotherapy for treatment of rheumatoid arthritis (ADACTA): a randomised, double-blind, controlled phase 4 trial. Lancet (London, England) 381:1541-1550. https://doi. org/10.1016/S0140-6736(13)60250-0

5. Taira BR, Singer AJ, Thode HC Jr, Lee CC (2009) National epidemiology of cutaneous abscesses: 1996 to 2005. Am J Emerg Med 27:289-292. https://doi.org/10.1016/j.ajem.2008.02.02711

6. Vaska VL, Nimmo GR, Jones M, Grimwood K, Paterson DL (2012) Increases in Australian cutaneous abscess hospitalisations: 1999-2008. Eur J Clin Microbiol Infec Dis 31:93-96. https://doi.org/10.1007/s10096-011-1281-3

7. Garner JP, Meiring PD, Ravi K, Gupta R (2007) Psoas abscess-not as rare as we think? Colorectal Dis 9:269-274. https://doi.org/10.1111/j.1463-1318.2006.01135.x

8. Navarro López V, Ramos JM, Meseguer V, Arellano JLP, Serrano R, Ordonez MAG et al (2009) Microbiology and outcome of iliopsoas abscess in 124 patients. Medicine (Baltimore) 88:120-130. https://doi.org/10.1097/MD.0b013e31819d2748

9. Shields D, Robinson P, Crowley TP (2012) Iliopsoas abscess-a review and update on the literature. Int J Surg 10:466-469. https ://doi.org/10.1016/j.ijsu.2012.08.016

10. Gasparyan AY, Ayvazyan L, Blackmore H, Kitas GD (2011) Writing a narrative biomedical review: considerations for authors, peer reviewers, and editors. Rheumatol Int 31:14091417. https://doi.org/10.1007/s00296-011-1999-3

11. Liu AY (2020) Infectious implications of Interleukin-1, Interleukin-6, and T helper type 2 inhibition. Infect Dis Clin North Am 34:211-234. https://doi.org/10.1016/j.idc.2020.02.003

12. Koike $T$, Harigai $M$, Inokuma $S$, Ishiguro $N$, Ryu J, Takeuchi $T$ et al (2014) Effectiveness and safety of tocilizumab: postmarketing surveillance of 7901 patients with rheumatoid arthritis in Japan. J Rheumatol 41:15-23

13. Nishimoto N, Ito K, Takagi N (2010) Safety and efficacy profiles of tocilizumab monotherapy in Japanese patients with rheumatoid arthritis: meta-analysis of six initial trials and five long-term extensions. Mod Rheumatol 20:222-232. https://doi. org/10.1007/s10165-010-0279-5

14. Emery P, Rondon J, Parrino J, Lin Y, Pena-Rossi C, van Hoogstraten $\mathrm{H}$ et al (2019) Safety and tolerability of subcutaneous sarilumab and intravenous tocilizumab in patients with rheumatoid arthritis. Rheumatology (Oxford) 58:849-858. https://doi. org/10.1093/rheumatology/key361

15. Sakai R, Cho S-K, Nanki T, Watanabe K, Yamazaki H, Tanaka $\mathrm{M}$ et al (2015) Head-to-head comparison of the safety of tocilizumab and tumor necrosis factor inhibitors in rheumatoid arthritis patients (RA) in clinical practice: results from the registry of Japanese RA patients on biologics for long-term safety (REAL) registry. Arthritis Res Ther 17:74. https://doi.org/10.1186/s1307 5-015-0583-8

16. Tsujimoto T, Takahata M, Kokabu T, Matsuhashi M, Iwasaki N (2016) Pyogenic spondylodiscitis following anti-interleukin-6 therapy in a patient with rheumatoid arthritis (implication of hematogenous infection risk in degenerative intervertebral discs): a case report and review of the literature. J Orthop Sci 21:694-697. https://doi.org/10.1016/j.jos.2015.06.018

17. Sampogna G, Tessitore N, Bianconi T, Leo A, Zarbo M, Montanari E (2020) Spinal cord dysfunction after COVID-19 infection. Spinal Cord Ser Cases 6:92. https://doi.org/10.1038/ s41394-020-00341-x

18. Douglass E, Mondy K, Huth RG (2016) Salmonella epidural abscess in a patient with rheumatoid arthritis treated with tocilizumab. Infect Dis ClinPract 24:e7-e8. https://doi.org/10.1097/ IPC.0000000000000320

19. Yamate R, Matono T, Yaguchi T, Fujii Y, Goto Y, Tobino K et al (2019) Disseminated nocardiosis with Nocardia brasiliensis bacteremia in a patient with rheumatoid arthritis using tocilizumab. J Infect Chemother 25:552-555. https://doi.org/10.1016/j. jiac.2019.02.005

20. de Kruif MD, van Gorp ECM, Bel EH, Gerlag DM, Kunst PW (2012) Streptococcal lung abscesses from a dental focus following tocilizumab: a case report. ClinExpRheumatol 30:951-953

21. Zhou S, Qiao J, Bai J, Wu Y, Fang H (2018) Biological therapy of traditional therapy-resistant adult-onset Still's disease: an evidence-based review. TherClin Risk Manag 14:167-171. https:// doi.org/10.2147/TCRM.S155488

22. van de Sande MGH, van Slobbe-Bijlsma ER (2012) Necrotizing fasciitis in a rheumatoid arthritis patient treated with tocilizumab. Rheumatology (Oxford) 51:577-578. https://doi.org/10.1093/ rheumatology/ker336

23. Ogata A, Mori M, Hashimoto S, Yano Y, Fujikawa T, Kawa M (2010) Minimal influence of tocilizumab on IFN-gamma synthesis by tuberculosis antigens. Mod Rheumatol 20:130-133. https://doi. org/10.1007/s10165-009-0243-4

24. Manabe S, Yanagi H, Ozawa H, Takagi A (2017) Necrotising soft tissue infection without systemic toxicity in a patient with rheumatoid arthritis treated with tocilizumab. BMJ Case Rep 2017:bcr2017222826. https://doi.org/10.1136/bcr-2017-222826

25. Wallis D (2014) Infection risk and biologics. CurrOpinRheumatol 26:404-409. https://doi.org/10.1097/BOR.0000000000000072

26. Nishimoto $\mathrm{N}$, Kishimoto $\mathrm{T}$ (2006) Interleukin 6: from bench to bedside. Nat ClinPractRheumatol 2:619-626. https://doi. org/10.1038/ncprheum0338

27. Furst DE, Keystone EC, So AK, Braun J, Breedveld FC, Burmester GR et al (2013) Updated consensus statement on biological agents for the treatment of rheumatic diseases, 2012. Ann Rheum Dis 72(Suppl 2):ii2-ii34. https://doi.org/10.1136/annrheumdi s-2013-203348

28. Lortholary O, Fernandez-Ruiz M, Baddley JW, Manuel O, Mariette X, Winthrop K et al (2020) Infectious complications of rheumatoid arthritis and psoriatic arthritis during targeted and biological therapies: a viewpoint in 2020. Ann Rheum Dis 79:1532-1543. https://doi.org/10.1136/annrheumdis-2020-21709 2

29. Strangfeld A, Eveslage M, Schneider M, Bergerhausen HJ, Klopsch T, Zink A et al (2011) Treatment benefit or survival of the fittest: what drives the time-dependent decrease in serious infection rates under TNF inhibition and what does this imply for the individual patient? Ann Rheum Dis 70:1914-1920. https://doi. org/10.1136/ard.2011.151043

30. Doran MF, Crowson CS, Pond GR, O'Fallon WM, Gabriel SE (2002) Predictors of infection in rheumatoid arthritis. Arthritis Rheum 46:2294-2300. https://doi.org/10.1002/art.10529

31. Galloway JB, Hyrich KL, Mercer LK, Dixon WG, Fu B, Ustianowski AP et al (2011) Anti-TNF therapy is associated with an increased risk of serious infections in patients with rheumatoid arthritis especially in the first 6 months of treatment: updated results from the British Society for Rheumatology Biologics Register with special emph. Rheumatology 50:124-131. https://doi. org/10.1093/rheumatology/keq242

32. Malaviya A, Haroon N (2011) Infections associated with the use of biologic response modifiers in rheumatic diseases: a critical appraisal. Indian J Rheumatol 6:99-112. https://doi.org/10.1016/ S0973-3698(11)60040-X 
33. Grøn KL, Arkema EV, Glintborg B, Mehnert F, Østergard M, Dreyer L et al (2019) Risk of serious infections in patients with rheumatoid arthritis treated in routine care with abatacept, rituximab and tocilizumab in Denmark and Sweden. Ann Rheum Dis 78:320-327. https://doi.org/10.1136/annrheumdis-2018-214326

34. Ozen G, Pedro S, England BR, Mehta B, Wolfe F, Michaud K et al (2019) Risk of serious infection in patients with rheumatoid arthritis treated with biologic versus nonbiologic disease-modifying antirheumatic drugs. ACR Open Rheumatol 1:424-432. https ://doi.org/10.1002/acr2.11064

35. Angelini J, Talotta R, Roncato R, Fornasier G, Barbiero G, Dal Cin $\mathrm{L}$ et al (2020) JAK-Inhibitors for the treatment of rheumatoid arthritis: a focus on the present and an outlook on the future. Biomolecules 10:1002. https://doi.org/10.3390/biom10071002

36. Rajasimhan S, Pamuk O, Katz JD (2020) Safety of Janus kinase inhibitors in older patients: a focus on the thromboembolic risk. Drugs Aging 37:551-558. https://doi.org/10.1007/s40266-02000775-w

Publisher's Note Springer Nature remains neutral with regard to jurisdictional claims in published maps and institutional affiliations. 